\title{
Content- based Image Retrieval Approach using Three Features Color, Texture and Shape
}

\author{
Yogendra Kumar Jain \\ Head of Department \\ Computer Science \& Engineering Department \\ Samrat Ashok Technological Institute, Vidisha
}

\author{
Rahul Yadav \\ Research scholar \\ Computer Science \& Engineering Department \\ Samrat Ashok Technological Institute, Vidisha
}

\begin{abstract}
Content Based Image Retrieval is a technique of automatic indexing and retrieving of images from a large data base. Visual features such as color, texture and shape are extracted to differentiate images in Content Based Image Retrieval (CBIR). Each of the features can be represented using one or more feature descriptors. These features descriptors combined with form feature vectors and are used together. During the retrieval, features and descriptors of the query are compared with the available images in the database. The images are then retrieved from database on the basis of distance of their feature vectors. At present, information of the maximum two features have been utilized for comparing the image and these methods provides the less accurate result. In our proposed work, more than two features i.e. three features are used for comparison and retrieval of image from the database. These three features are color, shape \& texture features for image retrieval and provide more accurate results. These features are combined to fulfil the aspect of retrieval in image. The proposed work uses HSI color information especially Hue value, Fuzzy C-Mean algorithm for shape representation and co-occurrence matrix is used for texture feature extraction.
\end{abstract}

\section{Keywords}

Color Histogram, FCM Algorithm, GLCM, Feature Vector (FV), CBIR.

\section{INTRODUCTION}

The Explosive growth of digital libraries due to web cameras, digital cameras, and mobile phones equipped with such devices is making the database management by human annotation an extremely tedious and clumsy task. Thus, there exists a dire need for developing an efficient expert technique that can automatically search the desired image from the huge database. To do this, two main approaches had been developed: textual image retrieval and content-based image retrieval. Textual image retrieval depends on attaching textual description, captioning or metadata. Then the query keywords in their metadata are used to retrieve images. However, in order to be able to use the image metadata, all digital images must be annotated with the metadata. Manual annotation requires people to have a look at each image and annotate it accordingly. Since manual annotation of large image databases is both expensive and time consuming. The text descriptions for each record in a database are created after laborious and time-consuming human intervention. The problems of text-based search are well-known: very large number of results, very large number of not relative results, difficulty in using suitable keywords and restriction of use of the language in which the system is built. To overcome the limitations of the keyword-based approach, new tools and interaction paradigms have been developed to search for visual information by referring directly to its content i.e. Content-Based Image Retrieval (CBIR). The visual elements such as color, texture, shape, structure, and spatial relationships have been used as clues for retrieving for example database images with similar content [1] [2]. Then relevant images are retrieved by comparing the low-level features of each item in the database with those of a usersupplied sketch, or more often, a key-image that is either selected from a restricted image set or is supplied by the user (query-by-example). This paradigm requires that the user provide a prototype image which is used as a reference example so as to express perceptual aspects of low/ intermediate features of visual content.

A typical CBIR system is an image retrieval system which includes three major aspects: high-dimensional indexing, feature extraction, and system design [3]. Among the three aspects, feature extraction is the basis of CBIR. However, features one can extract from the data are often low-level features. As a result, two semantically similar objects may lie far from each other in the feature space, while two completely different objects may stay close to each other. Although many features have been designed for general or specific CBIR systems, and some of them provides good retrieval performance.

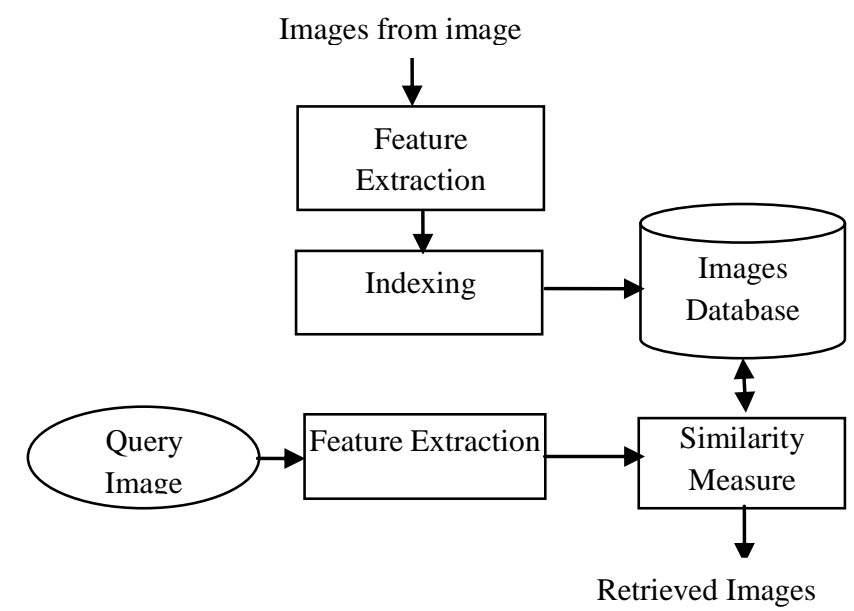

Fig. 1: Typical content-based image retrieval scenario

This paper focus on a broad domain of images: outdoor and indoor scenes, tribal people, beaches, buses, landscapes, cityscapes, plants, animals, objects, etc. A challenging aspect of our work is that it attempt to accomplish both feature extraction and segmentation. There are two main goals in proposed work.

1. The first is to develop a segmentation algorithm for images of innate scenes, in which texture and color 
usually do not exhibit uniform statistical characteristics.

2. The second is to incorporate knowledge of human perception in the design of underlying feature extraction algorithms.

\section{LITRATURE REVIEW}

Initially query-by-example scheme has been realized for the query by image content system [1]. Newer contributions to query-by-example include UCSB's Netra [2], UIUC's Mars [3], MIT's Photobook [4], Columbia's Visual SEEK [5], and ITI's Istorama [6]. The general framework of query-byexample have employed by them, demonstrating the use of various indexing feature-sets either in the image or in the region domain. The main drawback of all these retrieval system is that, in order to start a query, the availability of an appropriate key-image is assumed; occasionally, this is not feasible, particularly for image classes that are underrepresented in the database. Shih et. al. proposed a method using signature of the object, with Object Normalization and Detection/Separation, the Shape Representations and Similarity Measurement with affine invariants were implemented [7].

Image segmentation is a vital task in many research fields including computer vision [20] and intelligent image and video analysis [21]. Its essential goal is to split the pixels of an image into a set of regions such that the pixels in the same region are homogeneous according to some properties and the pixels in different regions are not similar. Segmentation of image found to be more successful in extracting low-level features in an image. Further Zhang et. al. proposed an issue for effective and efficient content based image retrieval by presenting a novel indexing and retrieval methodology that combines color, shape and texture, information for the indexing and retrieval [8]. Clustering, particularly fuzzy Cmeans (FCM)-based clustering and its variants, have been extensively used for segmentation due to their simplicity and fast convergence [20] [21] [22] [23]. By carefully selecting input features such as intensity, pixel color, texture, or a grouping of these data, the FCM algorithm can segment images to numerous regions.

Portilla and Simoncelli [9] proposed a statistical model for texture images that is reliable with human acuity. Their model is quite elaborative and captures a very wide class of textures. The texture analysis has been extensively used in computer vision and pattern recognition applications due to its capability of extracting the prominent features. Moghaddam et. al. introduced the concept of wavelet correlogram [10] and shown that the performance improvement can be obtained by optimizing the quantization thresholds [11] using genetic algorithm for CBIR application. Texture retrieval is a limb of texture analysis that has attracted wide attention from industries since this is well suited for the identification of products such as ceramic tiles, marble, parquet slabs, etc. Ahmadian et al. have used the discrete wavelet transform (DWT) for texture classification [12]. The application of the DWT using generalized Gaussian density with KullbackLeibler distance has shown to provide efficient results for texture image retrieval [13] and image segmentation [14]. However, the DWT can extract only three directional (horizontal, vertical, and diagonal) information from an image. To address this directional limitation, Gabor transform (GT) [15], rotated wavelet filters [16], dual tree complex wavelet filters (DT-CWFs), DT rotated CWFs [17], and rotational invariant complex wavelet filters [18] have been proposed for texture image retrieval. Wang et. al. [19] presents an effective color image retrieval method focus on texture, which uses the color co-occurrence matrix to extract the texture feature and measure the similarity of two color images.

\section{PROPOSED SYSTEM APPROACH}

The proposed method uses a texture representation method for image retrieval based on GLCM and fuzzy c-means is used to extract the shape features of an image, as described briefly as follows:

\begin{tabular}{|c|c|c|l|}
\hline \multicolumn{4}{|c|}{ Components of proposed approach } \\
\hline No. & Type & Method & \multicolumn{1}{c|}{ Description } \\
\hline 1. & Color & $\begin{array}{c}\text { Color } \\
\text { Histogram }\end{array}$ & used to extract color features \\
\hline 2. & Texture & GLCM & used to extract texture features \\
\hline 3. & Shape & FCM & used to extract shape features \\
\hline 4. & Similarity & $\begin{array}{c}\text { Euclidean } \\
\text { measure }\end{array}$ & $\begin{array}{l}\text { used to calculate the distance } \\
\text { between FV of Query image and } \\
\text { database image }\end{array}$ \\
\hline
\end{tabular}

When a user inputs a query image, retrieval system extracts features by applying pertinent algorithms as discussed in the consequent sections. The features extracted from images are stored in a feature vectors. After that, a similarity measure function based on Euclidean distance applied to produce final output relevant to the given query image as shown in Fig. 2.

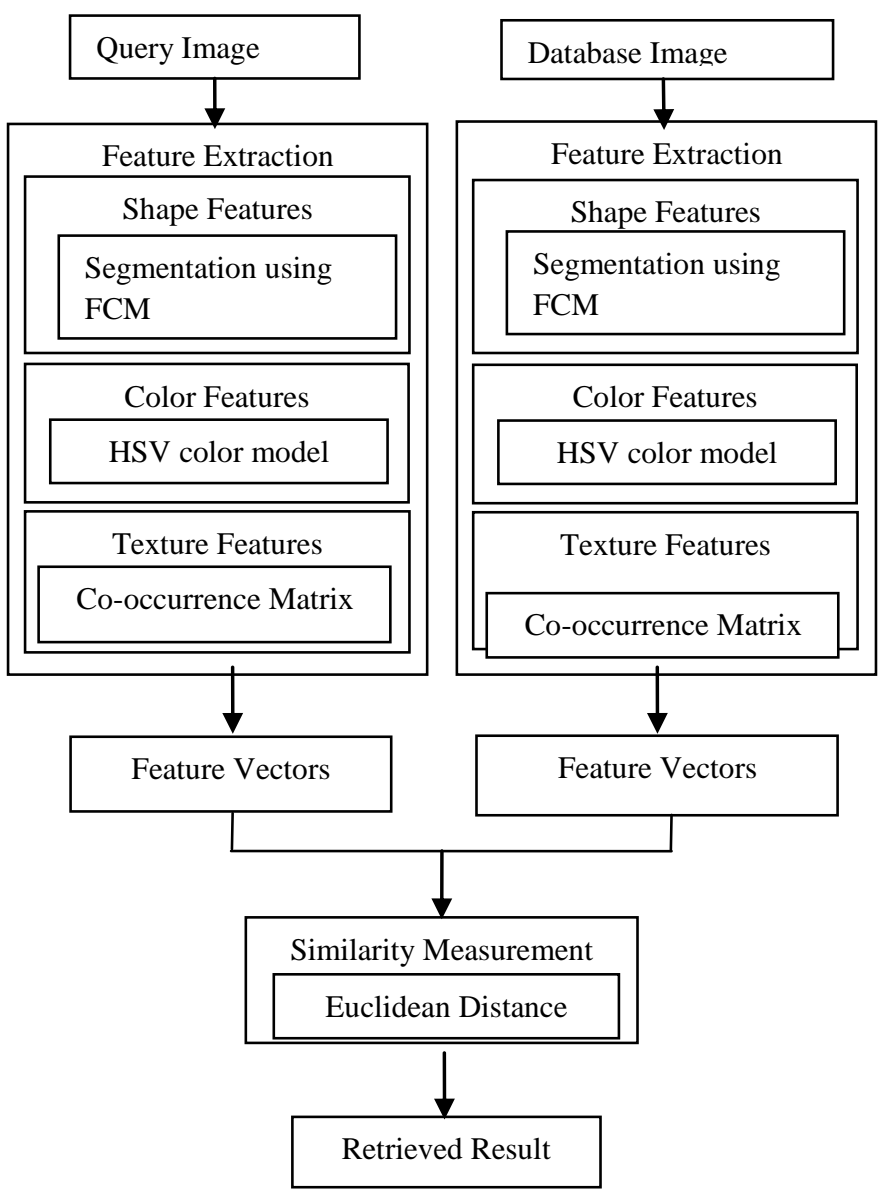

Fig. 2: Proposed content-based image retrieval approach 


\subsection{TEXTURE FEATURE}

Image texture refers to surface patterns which show gritty details of an image. It also provides discriminatory information regarding to the association of different colors, for example, as different patterns can be seen in grass fields and block walls. That makes them different from each other. There exist two main approaches for texture analysis. They are statistical and structural approaches [10]. In structural based texture method, the surface pattern is repetitive such as floor design that contains the same pattern [11]. In statistical texture, the surface pattern is not regularly repetitive such as different flower objects in a picture that normally have similar properties but not exactly the same [12]. The common known texture descriptors are Wavelet Transform, Gabor-filter, cooccurrence matrices and Tamura features. Co-occurrence matrix is a popular representation of texture feature of an image. The texture of image is a representation of spatial relationship of gray level image [49]. Co-occurrence matrix is constructed based on the orientation and distance between image pixels. Texture information can be extracted from image by Gray Level co-occurrence matrix. There are various texture features that can be extracted from an image using cooccurrence matrix, identified as Entropy, Correlation, Contrast, Energy, Inverse Difference Moment, Homogeneity etc. These features represent image as texture features and these texture features can be used for image retrieval purposes. Texture is another important feature of an image that can be extracted for the purpose of image retrieval. The second element of our proposed approach is the texture feature. For this purpose, the GLCM algorithm is used.

Haralick proposed Gray Level Co-occurrence Matrix (GLCM) method in 1973, which is based on the conditional probability density function. The co-occurrence matrix is a second order statistical measurement as it calculates the relationship between two pixels. To formulate the co-occurrence matrix, assume that the area to be analysed for texture information is a rectangular window of $\mathrm{M} \times \mathrm{N}$, and the grey level in each pixel is quantized to Gn levels. Co-occurrence matrix is a function of direction and distance. In a specified direction and distance, which can compute the symbiotic greyscale pixel i and $j$, given as the number of co-occurrence matrix element $p$ $(\mathrm{i}, \mathrm{j} \mid \mathrm{d}, \theta)$

$$
p(i, j \mid d, \theta)=\frac{p(i, j \mid d, \theta)}{\sum_{i} \sum_{j} p(i, j \mid d, \theta)}
$$

The matrix $P(i, j)$ is normalized by dividing each element in the matrix by the total number of pixel pairs. At first the cooccurrence matrix is constructed, on the basis of the distance and direction between image pixels. Then meaningful statistics can be extracted from the matrix as the texture representation. The texture features such as entropy, energy, contrast, and homogeneity. There are several common texture measures that can be extracted from the co-occurrence matrix of gray levels of an image, among which four parameters are mainly used as following:

(1) Moment of inertia (contrast):

$$
\mathrm{I}=\sum_{\mathrm{i}} \sum_{\mathrm{j}}(\mathrm{i}-\mathrm{j})^{2} \mathrm{P}(\mathrm{i}, \mathrm{j})
$$

Image contrast is the measure of sharpness of the image: the deeper grooves of the image texture, the greater the contrast is.

$$
\begin{aligned}
& \text { (2) Energy: } \\
& \quad \mathrm{E}=\sum_{\mathrm{i}} \sum_{\mathrm{j}}[\mathrm{P}(\mathrm{i}, \mathrm{j})]^{2}
\end{aligned}
$$

Energy is the measure of gray distribution uniformity of image. The coarser the texture is, the more energy it contains.

(3) Entropy:

$$
H=\sum_{i} \sum_{j}[P(i, j)] \log P(i, j)
$$

Entropy is a measure of the amount of information of an image. Entropy associates to the texture information. If there is no texture information, the entropy is Null

(4) Correlation:

$$
\mathrm{C}=(\mathrm{d}, \theta)=\frac{\sum_{\mathrm{i}, \mathrm{j}}\left(\mathrm{i}-\mu_{\mathrm{x}}\right)\left(\mathrm{j}-\mu_{\mathrm{y}}\right) \mathrm{P}(\mathrm{i}, \mathrm{j})}{\sigma_{\mathrm{x}} \sigma_{\mathrm{y}}}
$$

Correlation is a statistical measure that indicates the degree of resemblance of elements in GLCM.

$$
\begin{aligned}
& \text { Where } \mu_{\mathrm{x}}=\sum_{\mathrm{i}} \sum_{\mathrm{j}} \mathrm{iP}(\mathrm{i}, \mathrm{j}), \mu_{\mathrm{y}}=\sum_{\mathrm{i}} \sum_{\mathrm{j}} \mathrm{j} \mathrm{P}(\mathrm{i}, \mathrm{j}) \\
& \sigma_{\mathrm{x}}=\sum_{\mathrm{i}} \sum_{\mathrm{j}}\left(\mathrm{i}-\mu_{\mathrm{x}}\right)^{2} \mathrm{P}(\mathrm{i}, \mathrm{j}), \sigma_{\mathrm{y}}=\sum_{\mathrm{i}} \sum_{\mathrm{j}}\left(\mathrm{j}-\mu_{\mathrm{y}}\right)^{2} \mathrm{P}(\mathrm{i}, \mathrm{j})
\end{aligned}
$$

(5) Texture similarity measure:

Given image Q, I, the difference between the texture features are defined as:

$$
\mathrm{S}_{\mathrm{t}}(\mathrm{Q}, \mathrm{I})=1-\sum_{\mathrm{i}=1}^{4} \sum_{\mathrm{j}=1}^{4} \mathrm{w}_{\mathrm{ij}}\left|\mathrm{f}_{\mathrm{ij}}(\mathrm{Q})-\mathrm{f}_{\mathrm{ij}}(\mathrm{I})\right| \text {. }
$$

\subsection{COLOR FEATURE}

Color is one of the most significant features used for retrieval of images. There are numerous color models used to express Color features. HSV model is most consistent with the induction of the human visual model [4]. $\mathrm{H}$ corresponds to color hue, and it is the wavelength of the light reflected from an object or throughout the object; $\mathrm{S}$ corresponds to color saturation, which defines how much white is added to the color; V represents brightness (value), is the degree of color shading. a few representative color is extracted to represent image, thereby to reduce storage space and enhance the purpose of processing speed [5].

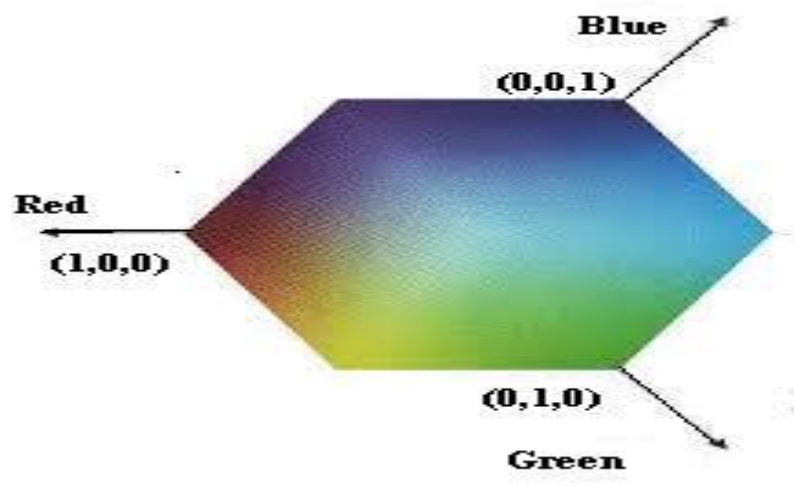

Fig. 3 RGB color-space

The HSV space color histogram used to describe the color feature of the overall image. According to human visual ability, hue $(\mathrm{H})$ is quantized into 16 bins, saturation( $\mathrm{S})$ is quantized into 4 bins and value (V) is quantized into 4 bins. Among those three components human cognitive about color is mainly based on hue, and then saturation, finally value. Then non-interval quantization based on different colors combined with the frequency bandwidth for each color, so quantized one-dimensional vector $\mathrm{L}$ is obtained:

$$
\mathrm{L}=16 \mathrm{H}+4 \mathrm{~S}+\mathrm{V}
$$

Where $\mathrm{L}$ is an integer between 0 and 255 
Image statistical histogram is represented as one-dimensional discrete function:

$$
\mathrm{h}_{\mathrm{k}}=\frac{\mathrm{n}_{\mathrm{k}}}{\mathrm{n}}, \mathrm{k}=0,1 \ldots \mathrm{L}-1
$$

The letter $\mathrm{k}$ represents Eigen values of color; letter $\mathrm{L}$ represents the number of feature vectors. Finally the color histogram of the image $\mathrm{I}$ is obtained as follows:

$$
\mathrm{H}_{\mathrm{i}}=\left[\mathrm{h}_{1}, \mathrm{~h}_{2}, \ldots . \mathrm{h}_{\mathrm{L}-1}\right]
$$

Histogram of an image is calculated to obtain color feature vector in HSV color-space.

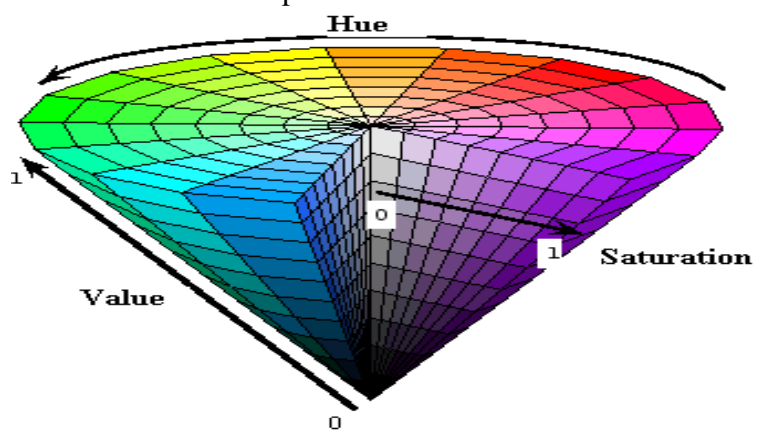

Fig. 4 HSV color-space

\subsection{SHAPE FEATURE}

Shape feature plays a vital role in object detection and identification. Shape features present robust and efficient information of objects in order to identify and distinguish them. Shape retrieval involves three primary issues: shape indexing, shape representation, and shape similarity. Among these, shape representation is the most concerned in shape retrieval. Various shape representation techniques, or shape descriptors, are present in the literature, these methods can be classified as: region based versus contour based. Contour based methods are usually extracting the boundary features of an object. These methods entirely disregard the significant features within the boundaries. Region-based methods rely up on shape descriptors are usually extract both kinds of features: boundary and region. Region-based image retrieval methods initially implement segmentation to split an image into dissimilar regions/segments, using threshold values according to the enviable results. On the other hand by applying any edge detection method to an image the boundary of an image can be obtained.

\subsubsection{Segmentation}

Image segmentation can be defined as the process of merging pixels having similar features into the same groups, or regions. The segmented image is then the union of distinct groups, where pixels of homogeneous regions are associated to the same groups. The proposed work focus on the fuzzy cmeans algorithm (FCM, [3]), which is used by many segmentation methods [1] [13] [5] [22] [12].

After successful segmentation, boundary of the extracted object is transformed into signature whose Fast Fourier Transform is calculated. FFT gives the array vector equivalent to the number of regions obtained after segmentation and is stored in database as first feature vector.

\section{Fuzzy C-mean Clustering Algorithm-}

This paper uses the (unsupervised) fuzzy C-means clustering (FCM) algorithm [14] which is an iterative procedure. Given $\mathrm{M}$ input feature vector $\mathrm{x}_{\mathrm{m}} ; \mathrm{m}=1 \ldots \mathrm{M}$, the number of clusters $\mathrm{C}$ where $2 \leq \mathrm{C}<\mathrm{M}$, and the fuzzy weighting exponent $\mathrm{z}, 1<$ $\mathrm{z}<\infty 1$, initialize the fuzzy membership function $\mathrm{u}_{\mathrm{c}, \mathrm{m}}^{(0)}(0)$ which is an entry of a $\mathrm{C} \times \mathrm{M}$ matrix $\mathrm{U}^{(0)}$. The following steps are iterated for increments of $b$ :

1) Calculate the fuzzy cluster centers $V_{c}^{b}$ with

2) Update U with

$$
\mathrm{V}_{\mathrm{c}}^{\mathrm{b}}=\frac{\sum_{\mathrm{m}=1}^{\mathrm{M}}\left(\mathrm{U}_{\mathrm{c}, \mathrm{m}}^{\mathrm{b}}\right)^{\mathrm{z}} \mathrm{X}_{\mathrm{m}}}{\sum_{\mathrm{m}=1}^{\mathrm{M}}\left(\mathrm{u}_{\mathrm{c}, \mathrm{m}}^{\mathrm{b}}\right)^{\mathrm{z}}}
$$

$$
\mathrm{U}_{\mathrm{c}, \mathrm{m}}^{\mathrm{b}+1}=\left[\sum_{\mathrm{j}=1}^{\mathrm{C}}\left\langle\frac{\alpha_{\mathrm{c}, \mathrm{m}}}{\alpha_{\mathrm{j}, \mathrm{m}}}\right)^{\frac{2}{2-1}}\right]^{-1}
$$

Where $\left(\alpha_{j, m}\right)^{2}=\left\|x_{m}-V_{j}^{b}\right\|^{2}$ and $\|$.$\| is any inner$ product induced norm.

3) Compare $U^{(b)}$ with $U^{(b+1)}$ is in a convenient matrix norm.

$$
\begin{gathered}
\text { If }\left\|\mathrm{U}^{(\mathrm{b}+1)}-\mathrm{U}^{\mathrm{b}}\right\|^{2} \leq \mathcal{E}\left(\varepsilon=10^{-5}\right) \text { Stop, otherwise } \\
\text { return to step 1 }
\end{gathered}
$$

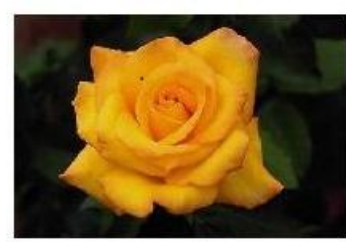

Original mage

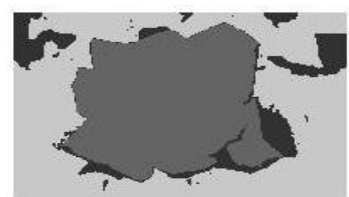

Segmented Image

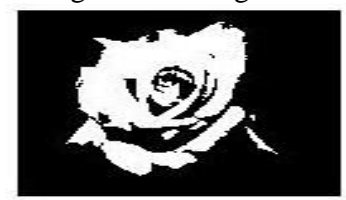

Region 2

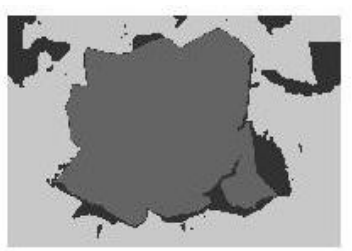

Segmented image using FCM

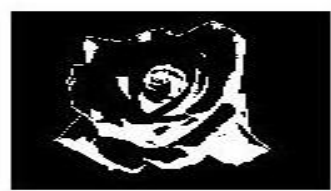

Region 1

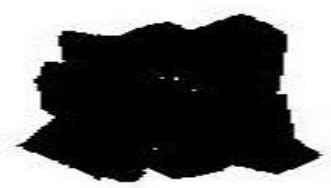

Region 3
Fig. 5: Region distribution of final segmentation result

\subsubsection{Shape signatures}

The signature of each region is developed by calculating distance between boundary elements and centroid of the region. The shape boundary coordinates $(x(t), y(t)), t=0$, 1 ... L-1, have been extracted in the pre-processing stage. The centroid distance function is expressed by the distance of the boundary points from the centroid $\left(\mathrm{x}_{\mathrm{c}}, \mathrm{y}_{\mathrm{c}}\right)$ of the shape

$$
r(t)=\left(\left[x(t)-x_{c}\right]^{2}+\left[x(t)-y_{c}\right]^{2}\right)^{\frac{1}{2}}
$$

Due to the subtraction of centroid, which signifies the position of the contour, from boundary coordinates, the centroid distance depiction is also invariant to translation. After computing signature of the image the Fast Fourier transform of signatures is calculated. These FFT coefficients give the Fourier descriptors of the shape. These descriptors constitute the shape of the object in a frequency domain. The lower frequency descriptors include information about the general features of the shape, and the higher frequency descriptors include information about finer details of the shape. In order to assist the use of the Fast Fourier transform (FFT), the number of sampled points is chosen to be power-of-two integer. By changing the number of sampled points, the precision of the shape representation can be adjusted. The greater the number, the more details the shape is represented, consequently, the matching result will be more accurate. In 
disparity, a smaller number of sampled points reduces the precision of the results, but improves the efficiency. For length $\mathrm{N}$ input vector $\mathrm{x}$, the DFT is a length $\mathrm{N}$ vector $\mathrm{X}$, The FFT is calculated as:

$$
X(k)=\frac{1}{N} \sum_{n=1}^{N} x(n)^{\frac{-j 2 \pi n t}{N}}
$$

The coefficients $\mathrm{x}(\mathrm{k}), \mathrm{n}=0,1 \ldots \mathrm{N}-1$, are usually called Fourier descriptors (FD) of the shape, referred as FDn, $\mathrm{n}=0,1 \ldots \mathrm{N}-1$

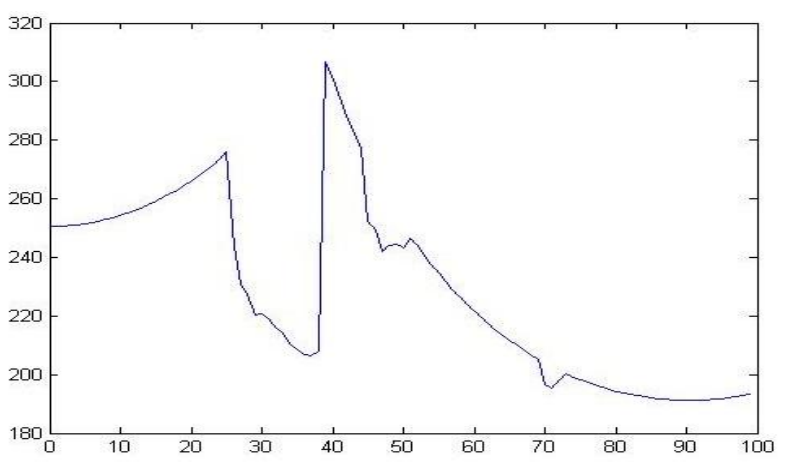

Fig. 6: Signature of segmented Image

\subsection{SIMILARITY MEASURE}

Similarity between two images is measured numerically that reflects the strength of connections between them. Similarity is crucial in obtaining relevant results. To obtain significant results different methods are used by researchers to evaluate similarity. For example, some researchers used fuzzy measures [9], histogram intersection [8, 24], and Euclidean distance [25]. In our proposed approach, Euclidean distance is used to compute the similarity between two feature vectors as follows

$$
\operatorname{ED}\left(M^{\mathrm{k}}, \mathrm{M}^{\mathrm{t}}\right)=\sqrt{\sum_{\mathrm{i}=1}^{\mathrm{n}}\left(\mathrm{M}_{\mathrm{i}}^{\mathrm{k}}-\mathrm{M}_{\mathrm{i}}^{\mathrm{t}}\right)^{2}}
$$

Mk and Mt represent image query and image database. Minimum distance represents the higher similarity between images.

\section{RESULT and ANALYSIS}

\subsection{Data Set Introduction}

In order to assess the performance of our proposed approach the same data-sets are used as used by the previous Researchers. The objective of this diverse collection is to evaluate the proposed approach thoroughly on different data sets so that Effectiveness and usefulness of the proposed approach can be demonstrated. Overall 10 categories of images have used for experiments. Each category of images contained 100 images such as African people, Buses, Flowers, Building, Horses, Dinosaur, Mountain and Food. The proposed method has been tested on a WANG database as shown in Fig. 7.

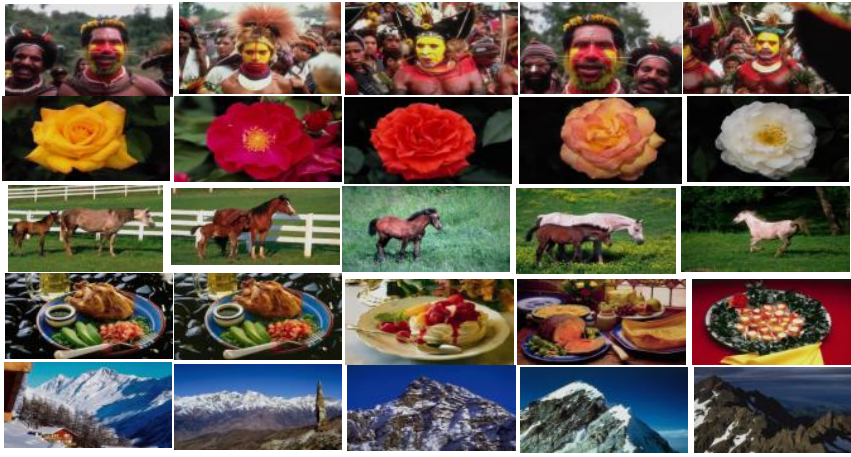

Fig. 7: Sample Images from Image Database

\subsection{Evaluation Criteria:}

Precision and Recall Curve: Generally the performance of the image retrieval is assessed in term of Average Precision (AP) and Average Recall (AR) [26]. The range of these two terms is $[0,1]$. In the analysis a high AR or AP value presents the better ability to retrieve the images and which is relevant. It shows intuitively the retrieval performance. They are defined as:

$$
\begin{aligned}
& \text { Precision }=\frac{\text { Number of relevant images retrieved }}{\text { Total number of images retrived }} \\
& \text { Recall }=\frac{\text { Number of relevant images retrieved }}{\text { Total number of relevant images }}
\end{aligned}
$$

The total number of items retrieved is the number of images that are returned by the search engine. The average precision of the images that belongs to the nth category (An) has been computed as

Where $n=1,2 \ldots \ldots 10$

$$
\mathrm{p}^{\prime \prime}=\sum_{\mathrm{j}=\mathrm{A}_{\mathrm{n}}} \frac{\mathrm{p}\left(\mathrm{i}_{\mathrm{j}}\right)}{\left|\mathrm{A}_{\mathrm{n}}\right|}
$$

Finally, the average precision is given by:

$$
\mathrm{p}^{\prime}=\sum_{\mathrm{n}=1}^{10} \frac{\mathrm{p}_{\mathrm{n}}^{\prime \prime}}{10}
$$

The average precision refers to the percentage of relevant images in top retrieved images and is calculated as the averaged precision values of all the queries to evaluate the effectiveness of the algorithm.

The average recall shows the fraction of the related images that are successfully retrieved and is defined as the percentage of retrieved images among all relevant images in the data set.

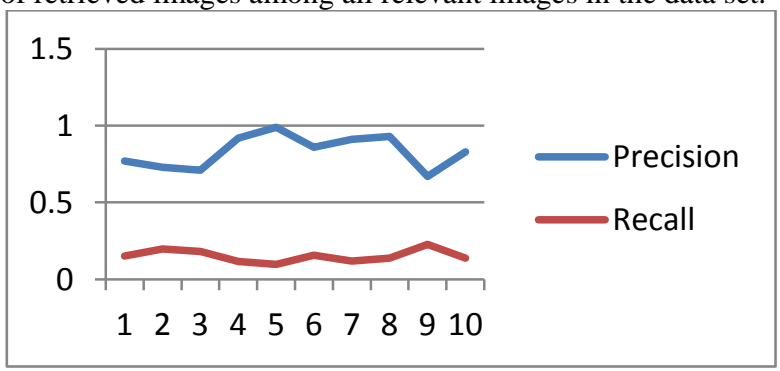

Fig. 8: Precision and Recall using proposed approach

\section{F-measure (F):}

It is a measure that combines precision and recall. It is also call the harmonic mean of precision and recall, basically used to measure the precision and recall for the fixed number of retrieved results. In our experiments, $\mathrm{F}$ is considers the first 20 retrieved models for the every query and calculate precision and recall over these results. The F-measure or balanced Fscore is expressed as $\mathrm{F}=2 * \mathrm{P} * \mathrm{R} /(\mathrm{P}+\mathrm{R})$. Where $\mathrm{P}$ and $\mathrm{R}$ is the precision and recall value for the results. 


\subsection{Discussion:}

A more quantitative evaluation is performed on 10 distinct image categories. In this paper randomly 15 images are choose from each category (i.e., 150 images in total) as query images and then calculate the average precision of each category by evaluating the top 20 returned results. Here, a retrieved image is considered as a correct match if and only if it is in the same category as the query image. The performance of the proposed image retrieval technique has been evaluated by comparing the results with the previous algorithm results as shown in the Table 2 . The effectiveness of the proposed retrieval method is evaluated by selecting 10 query images under each category. For each query image, the precision values of the retrieval results are analyzed based on the significance of the image semantics. The semantic appositeness is determined by manually comparing the query image and each of the retrieved images in the retrieval. The results are shown in Table 1 and Table 2 respectively. The 10 query retrievals by the proposed method are shown in Fig. 916. These results clearly show that the performance of the proposed method is better than the previous method.
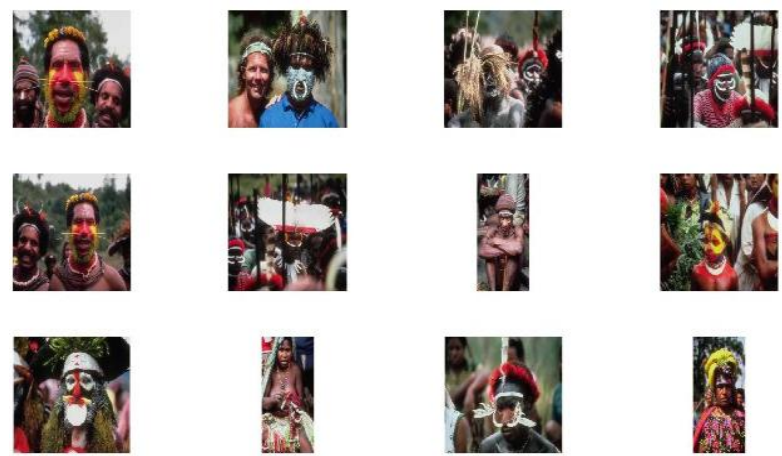

Fig. 9: Retrieve results for African People
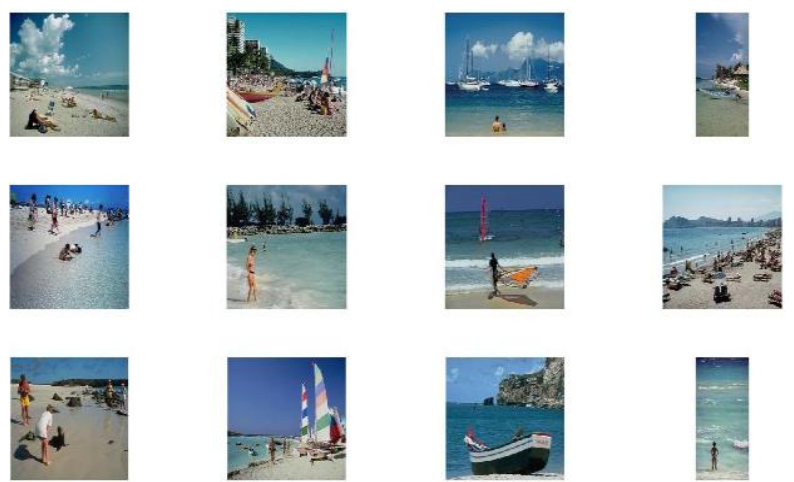

Fig. 10: Retrieve results for Beach
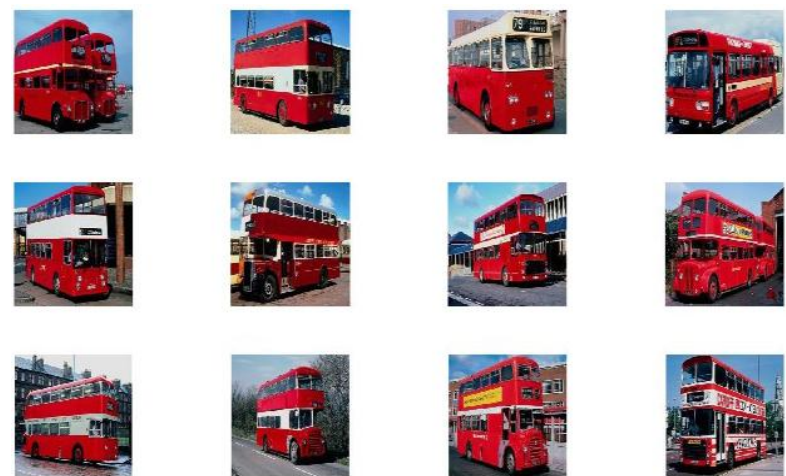

Fig. 11: Retrieve results for Bus


Fig. 12: Retrieve results for Dinosaurs
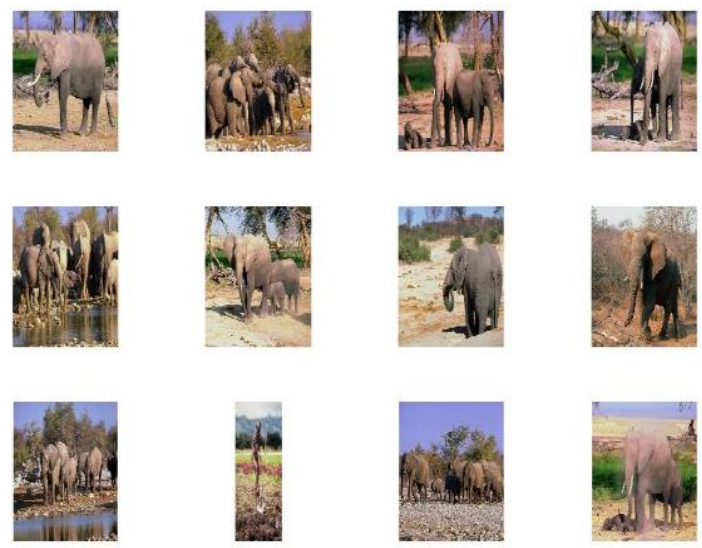

Fig. 13: Retrieve results for Elephants
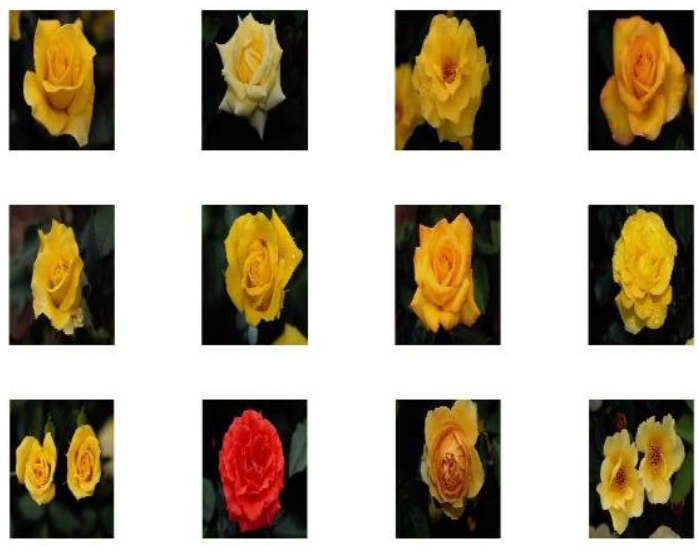

Fig. 14: Retrieve results for Flowers
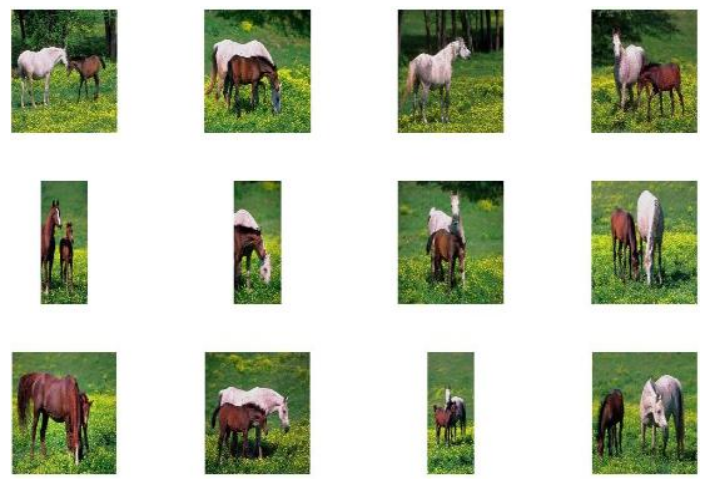
Fig. 15: Retrieve results for Horses
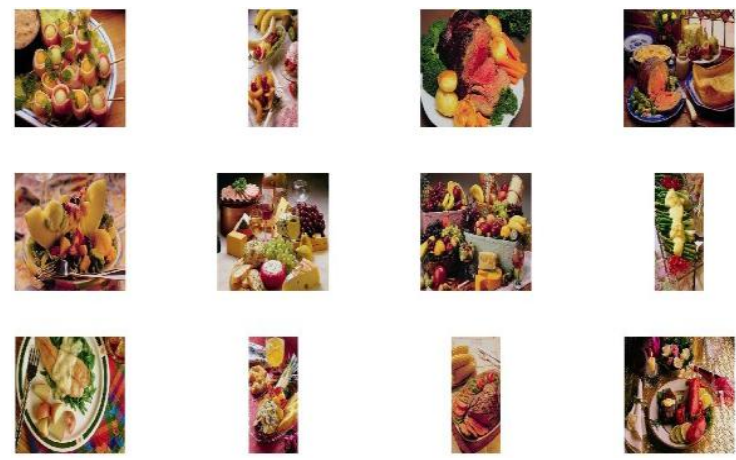

Fig. 16: Retrieve results for Food

Table1

Comparison of Images retrieval results with Precision

\begin{tabular}{|c|c|c|c|}
\hline Class & Category & $\begin{array}{c}\text { HSV + } \\
\text { FCM }\end{array}$ & $\begin{array}{c}\text { Proposed } \\
\text { method }\end{array}$ \\
\hline 1 & Tribal & 0.71 & 0.77 \\
\hline 2 & Beach & 0.67 & 0.73 \\
\hline 3 & Building & 0.62 & 0.71 \\
\hline 4 & Buses & 0.89 & 0.92 \\
\hline 5 & Dinosaurs & 0.93 & 0.99 \\
\hline 6 & Elephants & 0.73 & 0.86 \\
\hline 7 & Flowers & 0.83 & 0.91 \\
\hline 8 & Horses & 0.87 & 0.93 \\
\hline 9 & Mountains & 0.63 & 0.67 \\
\hline 10 & Food & 0.74 & 0.83 \\
\hline & $\begin{array}{c}\text { Average } \\
\text { Precision }\end{array}$ & $\mathbf{0 . 7 6 6}$ & $\mathbf{0 . 8 3 2}$ \\
\hline
\end{tabular}

Table 2

Comparison of Images retrieval results with Recall

\begin{tabular}{|c|c|c|c|}
\hline Class & Category & $\begin{array}{c}\text { HSV+ } \\
\text { FCM }\end{array}$ & $\begin{array}{c}\text { Proposed } \\
\text { method }\end{array}$ \\
\hline 1 & Tribal & 0.141 & 0.153 \\
\hline 2 & Beach & 0.189 & 0.198 \\
\hline 3 & Building & 0.173 & 0.182 \\
\hline 4 & Buses & 0.121 & 0.116 \\
\hline 5 & Dinosaurs & 0.101 & 0.098 \\
\hline 6 & Elephants & 0.147 & 0.156 \\
\hline 7 & Flowers & 0.113 & 0.118 \\
\hline
\end{tabular}

\begin{tabular}{|c|c|c|c|}
\hline 8 & Horses & 0.134 & 0.139 \\
\hline 9 & Mountains & 0.213 & 0.228 \\
\hline 10 & Food & 0.132 & 0.138 \\
\hline & $\begin{array}{c}\text { Average } \\
\text { Recall }\end{array}$ & $\mathbf{0 . 1 4 6}$ & $\mathbf{0 . 1 5 2}$ \\
\hline
\end{tabular}

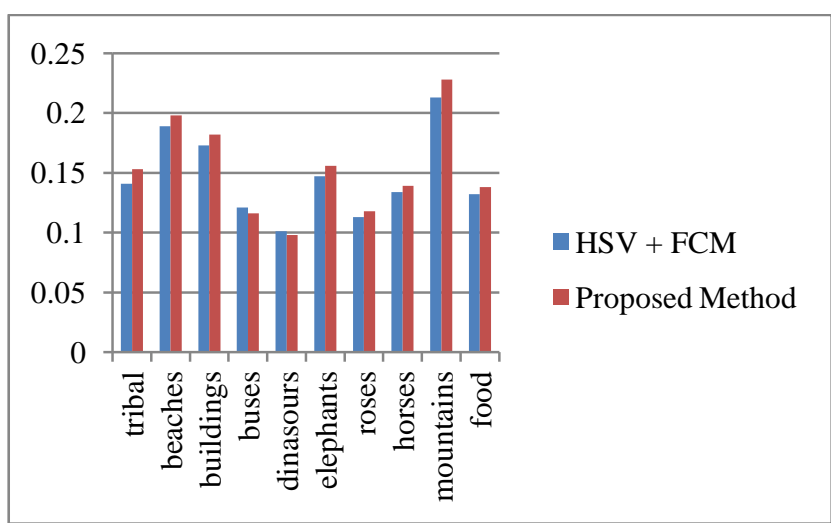

(a) Precision results

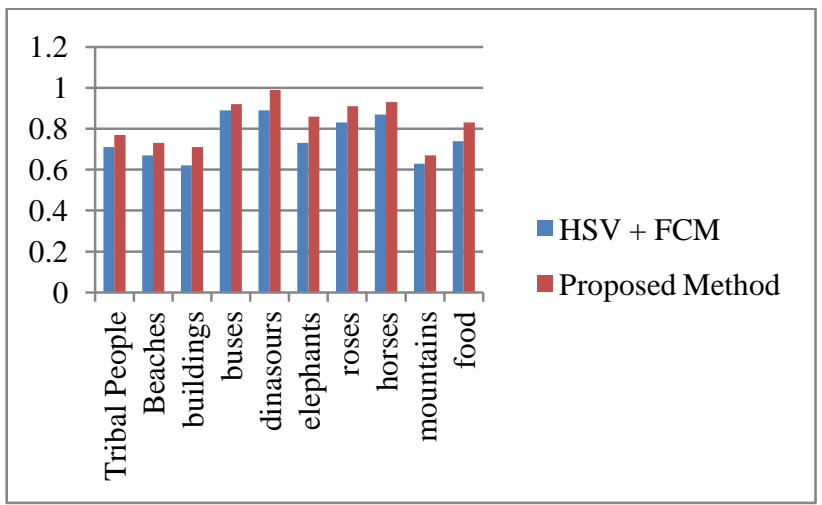

(b) Recall results

This paper has not included all the results of all queries in the paper due to space restriction, but they are integrated in Recall and Precision metrics in the next section. The results were randomly selected to be included in this paper (see Figs. 613). Our proposed approach effectively searches the similar images from huge available data-sets of images as can be seen in Figs. 6-13. The search is usually based on similarity rather than the exact match.

\section{CONCLUSION}

The proposed approach is based on the three well-known algorithms: color histogram, texture and image segmentation using FCM. The use of these algorithms assures that the proposed image retrieval approach present results which are extremely relevant to the content of query image. The color histogram is used to extract the color features of an image using four components such as Red, Green, Blue and Intensity. The co-occurrence matrix is used to extract the texture features and the FCM is used to extract the shape features of an image. The evaluation is carried out using the standard Precision and Recall measures, and the results are compared with the existing approaches. The presented results illustrate that the proposed approach provides improved results as compared to the existing 
methods. The presented results evidence the effectiveness and potential of our approach in the area of image retrieval. Future work entails applying the proposed method to real time surveillance data.

\section{REFERENCES}

[1] M. Flickner, H. Sawhney, W. Niblack, J. Ashley, Q. Huang, B. Dom, M. Gorkani, J. Hafner, D. Lee, Petkovic, D. Steele, and P. Yanker, "Query by image and video content: The QBIC system," Computer, vol. 28, no. 9, pp. 23-32, Sep. 1995.

[2] W. Y. Ma and B. S. Manjunath, "NeTra: A toolbox for navigating large image databases," Multimedia Syst., vol. 7, no. 3, pp. 184-198, 1999.

[3] T. S. Huang, S. Mehrotra, and K. Ramchandran, "Multimedia analysis and retrieval system (MARS) project," in Proc. 33rd Annu. Clinic on Library Application of Data Processing-Digital Image Access and Retrieval, Univ. Illinois at Urbana-Champain, 1996.

[4] A. Pentland, R. Picard, and S. Sclaro, "Photobook: Content-based manipulation of image databases," Int. J. Comput. Vis., vol. 18, no. 3, pp. 233-254, 1996.

[5] J. R. Smith and S.-F. Chang, "Visualseek: A fully automated content based image query system," ACM Multimedia, pp. 87-98, 1996.

[6] I. Kompatsiaris, E. Triantafyllou, and M. G. Strintzis, "Region-based color image indexing and retrieval," in Proc. IEEE Int. Conf. Image Processing, Thessaloniki, Greece, Oct. 2001.

[7] Timothy K. Shih, Lawrence Y. Deng, et. al. , Contentbased Image Retrieval with Intensive Signature via Affine Invariant Transformation, PP. 393-400.

[8] Ruofei Zhang, Zhongfei (Mark) Zhang, A Clustering Based Approach to Efficient Image Retrieval, Proceedings of the $14^{\text {th }}$ IEEE International Conference on Tools with Artificial Intelligence (ICTAI'02), 2002.

[9] J. Portilla and E. P. Simoncelli, "A parametric texture model based on joint statictics of complex wavelet coefficients," Int. J. Comput. Vis., vol. 40, pp. 49-71, Oct. 2000.

[10] H. A. Moghaddam, T. T. Khajoie, and A. H. Rouhi, "A new algorithm for image indexing and retrieval using wavelet correlogram," in Proc. ICIP, 2003, pp. III-497III-500.

[11] M. Saadatmand Tarzjan and H. A. Moghaddam, "A novel evolutionary approach for optimizing content based image retrieval," IEEE Trans. Syst., Man, Cybern. B, Cybern., vol. 37, no. 1, pp. 139-153, Feb. 2007.

[12] A. Ahmadian and A. Mostafa, "An efficient texture classification algorithm using gabor wavelet," in Proc. EMBS, 2003, pp. 930-933.

[13] M. N. Do and M. Vetterli, "Wavelet-based texture retrieval using generalized Gaussian density and
Kullback-Leibler distance,” IEEE Trans. Image Process., vol. 11, no. 2, pp. 146-158, Feb. 2002.

[14] M. Unser, "Texture classification by wavelet packet signatures," IEEE Trans. Pattern Anal.Mach. Intell., vol. 15, no. 11, pp. 1186-1191, Nov. 1993.

[15] B. S. Manjunath and W. Y. Ma, "Texture features for browsing and retrieval of image data," IEEE Trans. Pattern Anal. Mach. Intell., vol. 18, no. 8, pp. 837-842, Aug. 1996.

[16] M. Kokare, P. K. Biswas, and B. N. Chatterji, "Texture image retrieval using rotated wavelet filters," Pattern Recogn. Lett., vol. 28, no. 10, pp. 1240-1249, Jul. 2007.

[17] M. Kokare, P. K. Biswas, and B. N. Chatterji, "Texture image retrieval using new rotated complex wavelet filters," IEEE Trans. Syst., Man, Cybern. B, Cybern., vol. 35 , no. 6, pp. 1168-1178, Dec. 2005.

[18] M. Kokare, P. K. Biswas, and B. N. Chatterji, "Rotationinvariant texture image retrieval using rotated complex wavelet filters," IEEE Trans. Syst., Man, Cybern. B, Cybern., vol. 36, no. 6, pp. 1273-1282, Dec. 2006.

[19] Wang Xing-yuan, Chen Zhi-feng, Yun Jiao-jiao, An effective method for color image retrieval based on texture, Computer Standards \& Interfaces, vol. 34, pp. 31-35, 2012.

[20] W. L. Cai, S. C. Chen, and D. Q. Zhang, "Fast and robust fuzzy c-means clustering algorithms incorporating local information for image segmentation," Pattern Recognit., vol. 40, no. 3, pp. 825-838, Mar. 2007.

[21] K. Sikka, N. Sinha, P. K. Singh, and A. K. Mishra, "A fully automated algorithm under modified FCM framework for improved brain MR image segmentation," Magn. Reson. Imaging, vol. 27, no. 7, pp. 994-1004, Sep. 2009.

[22] K. S. Chuang, H. L. Tzeng, S. Chen, J. Wu, and T. J. Chen, "Fuzzy c-means clustering with spatial information for image segmentation," Comput. Med. Imaging Graph., vol. 30, no. 1, pp. 9-15, Jan. 2006.

[23] Y. A. Tolias and S. M. Panas, "Image segmentation by a fuzzy clustering algorithm using adaptive spatially constrained membership functions," IEEE Trans. Syst., Man, Cybern. A, Syst., Humans, vol. 28, no. 3, pp. 359369, May 1998

[24] A.M.W. Smeulders, M. Worring, S. Santini, A. Gupta, R. Jain, Content-based image retrieval at the end of the early years, IEEE Trans. Pattern Anal. Machine Intell. 22 (12) (2000) 1349-1380.

[25] F. Long, H. Zhang, D.D. Feng, Fundamentals of content-based image retrieval, in: Multimedia Information Retrieval and Management: Technological Fundamentals and Applications, Springer, 2003.

[26] V. Viitaniemi, J. Laaksonen, Evaluating the performance in automatic image annotation: Example case by adaptive fusion of global image features, Signal Process. Image Commun. 22 (6) (2007) 557-568. 